\title{
Durable Near-Complete Response to Anti-PD-1 Checkpoint Immunotherapy in a Refractory Malignant Solitary Fibrous Tumor of the Pleura
}

\author{
James T. Boothe $e^{a, b} \quad$ G. Thomas Budd ${ }^{c} \quad$ Matthew B. Smolkin ${ }^{a, d}$ \\ Patrick C. Ma ${ }^{a, b}, e$
}

aWVU Cancer Institute, Mary Babb Randolph Cancer Center, West Virginia University, Morgantown, WV, USA; bWVU School of Medicine, West Virginia University, Morgantown, WV, USA; 'C Cleveland Clinic Taussig Cancer Institute, Cleveland, OH, USA; ${ }^{\mathrm{d} D e p a r t m e n t}$ of Pathology, WVU Medicine Ruby Memorial Hospital, West Virginia University, Morgantown, WV, USA; eWest Virginia Clinical and Translational Science Institute, Morgantown, WV, USA

\section{Keywords}

Cancer immunotherapy - Checkpoint blockade - Solitary fibrous tumor of the pleura PD-L1 . PD-1

\begin{abstract}
Solitary fibrous tumor of the pleura is a rare and usually benign primary neoplasm arising from mesenchymal cells of the submesothelial tissue. We present here the case of a patient diagnosed with CD34-positive advanced malignant solitary fibrous tumor of the pleura whose disease failed to respond to combination cytotoxic chemotherapy agents, but demonstrated a prompt near-complete response to checkpoint blockade treatment using the antiprogrammed death (PD)-1 monoclonal antibody pembrolizumab, based on tumor molecular profiling revealing tumoral expression positivity for both programmed death-ligand 1 (PDL1) and PD-1. The patient experienced minimal adverse effects from the treatment with durable favorable response lasting up to cycle 26.




\section{Case Reports in Oncology}

Boothe et al.: Durable Near-Complete Response to Anti-PD-1 Checkpoint

Immunotherapy in a Refractory Malignant Solitary Fibrous Tumor of the Pleura

\section{Introduction}

Solitary fibrous tumor of the pleura (SFTP) is a rare primary neoplasm that arises from mesenchymal cells of the areolar tissue in the submesothelial layer of the pleura. It accounts for less than $5 \%$ of primary pleural neoplasms [1]. The neoplasm is usually benign, with reported malignancy accounting for up to $20 \%$ of cases. Surgical resection of SFTP usually yields a favorable outcome with a 5-year disease-free survival rate of $81 \%$ [2]. However, malignant SFTP has a $63 \%$ recurrence rate even following curative-intent complete surgical resection [3]. Due to the rarity of malignant SFTP, there is a striking lack of published literature on the efficacy of specific therapeutic regimens. Much focus has recently been directed by the clinical oncology community towards immune system-modulating therapies, including immune checkpoint blockade therapy [4]. One such approach involves administration of monoclonal antibodies with binding specificity to the programmed cell death protein 1 (PD1) expressed on the surface of T cells or the programmed death-ligand 1 (PD-L1) expressed on the surface of tumor cells $[5,6]$.

PD-L1 on healthy human cells binds to T-cell PD-1, initiating an inhibitory signaling cascade that has the effect of preventing development of a cytotoxic T-cell attack against the cells. We present the case of a patient with malignant SFTP whose disease failed to respond to several combination chemotherapy regimens but eventually demonstrated a durable near-complete response to checkpoint immunotherapy in the form of the anti-PD-1 monoclonal antibody pembrolizumab.

\section{Case Report}

A 50-year-old Caucasian male with a 30 pack-year smoking history, who was healthy with the only known medical issue being controlled hypertension, presented to the clinic with a sore lump over the right lower chest wall between the 6th and 7th ribs. Initial workup with CT chest imaging in December 2014 revealed a soft tissue mass arising within the right chest wall underlying the palpable lump. A follow-up CT of the chest, abdomen, and pelvis in January 2015 confirmed an enlarging underlying soft tissue mass arising within the chest wall soft tissue/pleural tissue space, centered over the intercostal space, measuring $3.0 \times 5.9$ $\times 5.0 \mathrm{~cm}$. An enlarged right mid-hilar lymph node superior to the right pulmonary artery measuring $2.7 \times 3.1 \times 2.7 \mathrm{~cm}$ was seen as well. Ultrasound-guided needle biopsy was performed on the chest wall mass, and subsequent pathological analysis, including a pathology review at the Mayo Clinic, revealed an extensively necrotic, poorly differentiated malignant neoplasm possibly representing a malignant SFTP (Fig. 1a). Immunohistochemistry analysis revealed that the neoplastic cells were diffusely positive for CD34 (Fig. 1b) and focally widespectrum cytokeratin-positive. INI-1 expression was retained. WT-1, calretinin, ER, CD31, ERG protein, FLI-1, and high MW cytokeratin were negative. PET/CT imaging in February 2015 showed no evidence of metastatic lesions in the abdomen, pelvis, or bones, and a bone scan was also negative for any distant metastases. The right lower chest wall mass was found intensely hypermetabolic with SUVmax 23, with the right hilar mass having SUVmax 20 (Fig. 2a-d). 


\section{Case Reports in Oncology}

The patient received front-line definitive concurrent chemoradiation treatment at a local cancer center using low-dose weekly carboplatin and paclitaxel and daily radiotherapy to a total dose of $64 \mathrm{~Gy}$. Restaging by PET/CT showed shrinkage of the primary chest wall mass to $2.8 \times 1.4 \mathrm{~cm}$ and persistence of the right hilar nodal mass. However, new soft tissue foci with FDG hypermetabolism were seen in the deep subcutaneous tissue of the left posterior abdominal wall at the level of the transverse process of the third vertebra, as well as two nodular foci at the pleural thickening of the right lung apex. Further consideration of surgical resection was canceled. Punch biopsy of the new subcutaneous mass revealed pathological characteristics similar to the previous biopsy, confirming metastatic dissemination. Based on this finding, a second-line palliative chemotherapy regimen of gemcitabine and docetaxel was initiated. However, after the first cycle of treatment, the patient suffered severe lifethreatening treatment-related toxicities, including neutropenic fever, pneumonia, severe fatigue, mouth sores, oral candidiasis, nausea/vomiting, decreased oral intake, and prerenal acute renal failure secondary to dehydration, requiring hospitalization. The chemotherapy regimen was aborted after successful treatment with antimicrobials and rehydration therapy in the hospital. The patient sought second opinion from a tertiary cancer institute, and was started on the MD Anderson regimen, which has reported efficacy for malignant SFTP in the literature, using temozolomide and bevacizumab (temozolomide $150 \mathrm{mg} / \mathrm{m}^{2}$ orally on days 1-7 and days 15-21, plus bevacizumab $5 \mathrm{mg} / \mathrm{kg}$ i.v. on days 8 and 22, repeated every 28-day cycle) [7]. However, after three cycles of this combination regimen, PET imaging in October 2015 showed further progression of the disease (Fig. 2e).

Genomic profiling analysis of the primary tumor using the FoundationHeme ${ }^{\circledR}$ assay revealed possible amplification of EGFR (equivocal), CDKN2A p16INK4a-R112P, TP53-V157F, CPS1-L878fs*8, and SMARCA4-E1364*. Since no definitive actionable genomic alterations were evident, we sought comprehensive multi-platform molecular and genomic profiling with the Caris Molecular Intelligence ${ }^{\circledR}(\mathrm{CMI}-\mathrm{X})$ test (Table 1) while the patient was undergoing temozolomide plus bevacizumab therapy. CMI-X tumor profiling confirmed positive results for PD-1 and PD-L1 (2+, 5\%; Fig. 1c) in immunohistochemistry assays. Temozolomide/bevacizumab treatment was eventually found ineffective soon thereafter.

Notably, the CMI-X molecular profiling also suggested potential lack of benefit to temozolomide therapy based on being MGMT-positive via IHC $(1+, 45 \%$; Table 1$)$. The key genomic alterations found in the next-generation sequencing (NGS) test include: TP53V157F (56\%), CDKN2A-R112P (62\%), and MLH1-E234Q (52\%). Altogether, these above findings prompted us to switch treatment strategy and ultimately we initiated treatment with an anti-PD-1 immune checkpoint monoclonal antibody in October 2015; pembrolizumab was selected for off-label use, using the FDA-approved dose regimen of $2 \mathrm{mg} / \mathrm{kg}$ intravenously every 3 weeks. When the patient returned for tumor response assessment in December 2015 after 2 cycles of treatment, he reported excellent tolerance and notable diminution of the left abdominal wall mass, which was confirmed as a prompt partial response by CT scan imaging (Fig. 2e). Restaging scan via CT imaging after the 5th cycle of pembrolizumab confirmed a near-complete response in February 2016. Prior to the planned 13th course of therapy, the patient reported no palpable masses, and restaging via CT scan imaging in July 2016 confirmed an ongoing persistent near-complete response with no new disease recurrence (Fig. 2e). To date, the patient has durable near-complete response after up to a total of 26 cycles of pembrolizumab infusions, and he remains well with excellent toler- 


\section{Case Reports in Oncology}

ance without significant adverse effects except for mild dry skin. The patient reported progressively improved energy throughout therapy, and his family reported that he regained his appetite and his ability to complete all tasks of daily living. He was found to have a new solitary left apical PET-avid pleural based mass biopsy-proven to be of similar histologic diagnosis as PD-1 therapy-acquired resistant disease after cycle 26. He received local radiotherapy to the isolated pembrolizumab-resistant tumor mass and continuation PD-1 immunotherapy with resultant ongoing response after cycle 31.

\section{Discussion}

Our case report highlights the substantial potential of PD-1/PD-L1 checkpoint pathway blockade in cancer immunotherapy. To date, the anti-PD-1 checkpoint antibodies nivolumab and pembrolizumab have been FDA-approved for the treatment of previously treated advanced melanoma and non-small cell lung cancer (NSCLC) [8-10]. Expansion of its use is evident in the growing list of tumor types that show treatment response with such treatment strategy, including classic Hodgkin lymphoma [11] and head and neck cancer [12]. While the role of PD-L1 tumoral expression as a predictive biomarker for anti-PD-1 immunotherapy remains to be better refined, it has been approved as a companion diagnostic in the pembrolizumab approval by the FDA. Pembrolizumab has also recently been approved as firstline therapy in advanced NSCLC patients whose tumor has strong PD-L1 expression as defined by tumor proportion score (TPS) $\geq 50 \%$, based on the positive survival data from the phase III KEYNOTE-024 study of single-agent pembrolizumab versus standard-of-care platinum-based doublet chemotherapy [13]. In May 2017, pembrolizumab received additional FDA approval as a first-line checkpoint inhibitor in combination with carboplatin and pemetrexed in advanced non-squamous NSCLC regardless of PD-L1 status [14]. A cancer type such as malignant SFTP is so rare that it is unrealistic to expect completion of phase III clinical studies to validate the treatment efficacy of immune checkpoint therapies. Thus, it is of practical value to observe and report remarkable responders in $N=1$ study or case reports. Our case study also illustrates that even with a low level of detected PD-L1 expression status of TPS 5\%, a prompt and ultimately durable remarkable near-complete response in chemotherapy- and radiation-refractory disease is possible. To our knowledge, this is the first report of a treatment response of a malignant SFTP to cancer checkpoint immunotherapy. Our PD-1 blockade strategy success serves as a reminder that the new paradigm of cancer immunotherapy can unleash significant anti-tumor effects even in heavily pretreated or chemotherapy- and radiation-refractory tumors. Moreover, other immune checkpoint inhibitors such as PD-L1 antibodies (e.g., atezolizumab, durvalumab/MEDI4736) or immunemodulating agents such as anti-cytotoxic T-lymphocyte-associated protein 4 (CTL4) antibodies (e.g., tremelimumab, ipilimumab) would also merit further studies to determine their potential efficacy in orphan diseases like SFTP. Further research in cancer immunotherapy predictive biomarkers is urgently necessary for optimizing patient selection for treatment. Undoubtedly, cancer immunotherapy has revolutionized modern cancer treatment and will continue to make further strides in improving cancer patients' treatment outcomes in future years. 
Boothe et al.: Durable Near-Complete Response to Anti-PD-1 Checkpoint

Immunotherapy in a Refractory Malignant Solitary Fibrous Tumor of the Pleura

\section{Acknowledgements}

This work was supported by IDeA CTR support - National Institute of Health (NIH)/National Institute of General Medical Sciences (NIGMS), U54GM104942 (P.C.M.). The content is solely the responsibility of the authors and does not necessarily represent the official views of the NIH. P.C.M. is supported by WVU Cancer Institute, and the West Virginia Clinical and Translational Science Institute (WVCTSI).

\section{Statement of Ethics}

The subject described in this case report has given his written informed consent for the publication of the report. The case study protocol has been approved by the Institution Review Board on human research.

\section{Disclosure Statement}

Speaker's bureau of Merck, Takeda-ARIAD (P.C.M.).

\section{References}

1 Lu C, Ji Y, Shan F, Guo W, Ding J, Ge D: Solitary fibrous tumor of the pleura: an analysis of 13 cases. World J Surg 2008;32:1663-1668.

2 Lococo F, Cesario A, Cardillo G, et al: Malignant solitary fibrous tumors of the pleura: retrospective review of a multicenter series. J Thorac Oncol 2012;7:1698-1706.

Robinson LA: Solitary fibrous tumor of the pleura. Cancer Control 2006;4:264-269.

Pardoll DM: The blockade of immune checkpoints in cancer immunotherapy. Nat Rev Cancer 2012;12:252-264.

5 Medina PJ, Adams VR: PD-1 pathway inhibitors: immuno-oncology agents for restoring antitumor immune responses. Pharmacotherapy 2016;36:317-334.

-6 Ma W, Gilligan BM, Yuan J, Li T: Current status and perspectives in translational biomarker research for PD-1/PD-L1 immune checkpoint blockade therapy. J Hematol Oncol 2016;9:47.

7 Park MS, Patel SR, Ludwig JA, et al: Activity of temozolomide and bevacizumab in the treatment of locally advanced, recurrent, and metastatic hemangiopericytoma and malignant solitary fibrous tumor. Cancer 2011;117:4939-4947. Herbst RS, Baas P, Kim DW, et al: Pembrolizumab versus docetaxel for previously treated, PD-L1positive, advanced non-small-cell lung cancer (KEYNOTE-010): a randomised controlled trial. Lancet 2016;387:1540-1550. Brahmer J, Reckamp KL, Baas P, et al: Nivolumab versus docetaxel in advanced squamous-cell nonsmall-cell lung cancer. N Engl J Med 2015;373:123-135.

10 Borghaei H, Paz-Ares L, Horn L, et al: Nivolumab versus docetaxel in advanced nonsquamous nonsmall-cell lung cancer. N Engl J Med 2015;373:1627-1639.

11 Ansell SM, Lesokhin AM, Borrello I, et al: PD-1 blockade with nivolumab in relapsed or refractory Hodgkin's lymphoma. N Engl J Med 2015;372:311-319.

$\checkmark 12$ Ferris RL, Blumenschein G, Fayette J, et al: Nivolumab for recurrent squamous-cell carcinoma of the head and neck. N Engl J Med 2016;375:1856-1867.

13 Reck M, Rodríguez-Abreu D, Robinson AG, et al: Pembrolizumab versus chemotherapy for PD-L1positive non-small-cell lung cancer. N Engl J Med 2016;375:1823-1833.

$\checkmark 14$ Langer CJ, Gadgeel SM, Borghaei H, et al: Carboplatin and pemetrexed with or without pembrolizumab for advanced non-small-cell lung cancer: a randomised, phase 2 cohort of the open-label KEYNOTE-021 study. Lancet Oncol 2016;17:1497-1508. 


\section{Case Reports in Oncology}
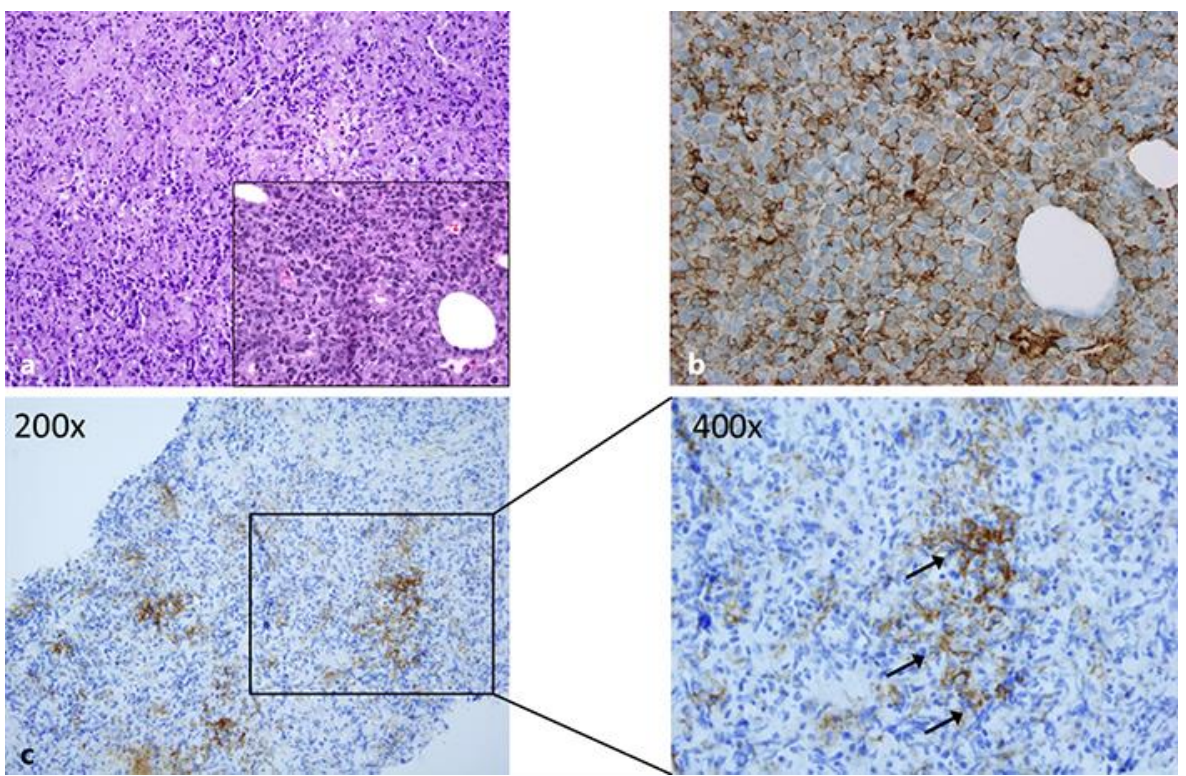

Fig. 1. Histopathologic features of the malignant solitary fibrous tumor of the pleura (SFTP). a A lowpowered view of hematoxylin and eosin staining, demonstrating the hypercellularity of the tumor (mag. $100 \times$ ) with the high-powered view demonstrating numerous mitoses shown in the inset (mag. 400x). b A high-powered view demonstrating positive immunohistochemical staining for CD34 expression of the SFTP (mag. 400×). c A low-powered view demonstrating positive immunohistochemical staining for PD-L1 expression of the SFTP (left, mag. 200x), with a corresponding high-powered view also shown (right, mag. $400 \times)$. 


\section{Case Reports in Oncology}

Boothe et al: Durable Near-Complete Response to Anti-PD-1 Checkpoint

Immunotherapy in a Refractory Malignant Solitary Fibrous Tumor of the Pleura
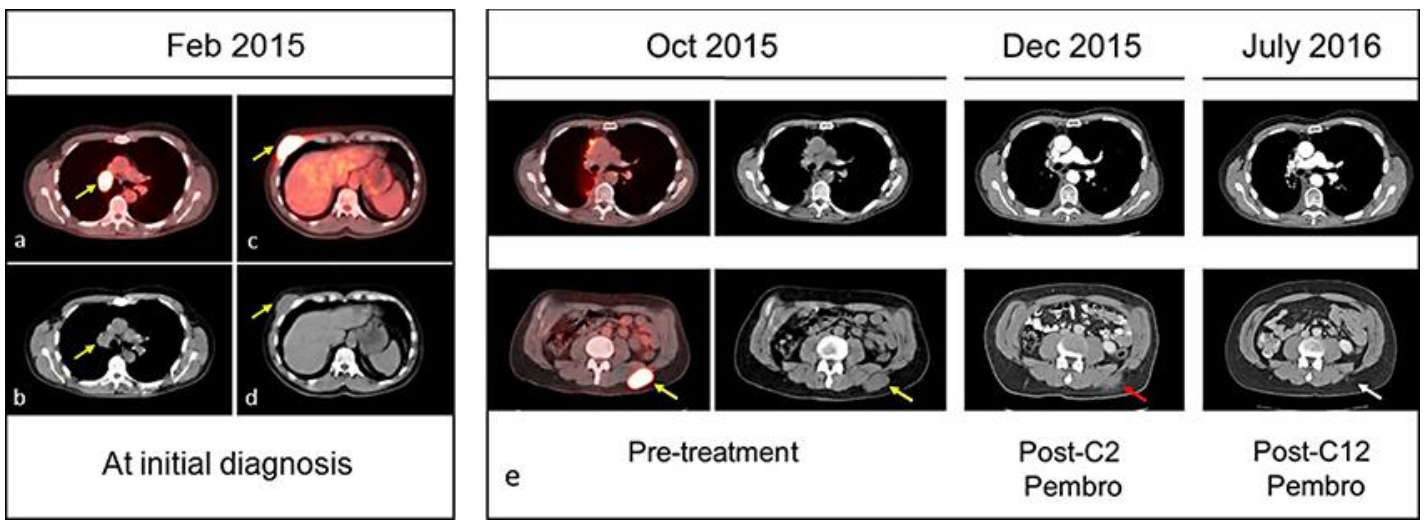

Fig. 2. Positron-emission tomography (PET)/computed tomography (CT) scan imaging of the patient with malignant solitary fibrous tumor of the pleura (SFTP) at the time of initial diagnosis and during PD-1 checkpoint inhibition response. Shown here are the selected sites of disease (yellow arrows) in the right hilar mass (a, b) and in the right lower chest wall mass (c, d) at the time of initial diagnosis. e Tumor response of the refractory malignant SFTP in the left posterior abdominal wall mass to PD-1-targeting checkpoint immunotherapy using pembrolizumab. Serial imaging in the pulmonary hilar region (top panels) and in the lower abdominal region (lower panels) during the course of treatment with pembrolizumab are shown here for illustration. Yellow arrows, pretreatment tumor burden; red arrow, early tumor response; white arrow, near-complete tumor response after pembrolizumab anti-PD-1 checkpoint immunotherapy. 


\section{Case Reports in Oncology}

Table 1. Comprehensive molecular tumor profiling with Caris Molecular Intelligence (CMI)-X assay

\begin{tabular}{|c|c|c|}
\hline Molecular-genomic alterations & $\begin{array}{l}\text { Therapy options with } \\
\text { potential benefits }\end{array}$ & $\begin{array}{l}\text { Therapy options with } \\
\text { potential lack of benefits }\end{array}$ \\
\hline TP53 exon 5 | V157F (56\%) & $\mathrm{n} / \mathrm{a}$ & $\mathrm{n} / \mathrm{a}$ \\
\hline CDKN2A exon 2 | R112P (62\%) & $\mathrm{n} / \mathrm{a}$ & $\mathrm{n} / \mathrm{a}$ \\
\hline MLH1 exon 9 | E234Q (52\%) & $\mathrm{n} / \mathrm{a}$ & $\mathrm{n} / \mathrm{a}$ \\
\hline PTEN IHC - positive & $\mathrm{n} / \mathrm{a}$ & $\mathrm{n} / \mathrm{a}$ \\
\hline TS IHC - positive $(1+75 \%)$ & $\mathrm{n} / \mathrm{a}$ & $\begin{array}{l}\text { Capecitabine, fluorouracil, } \\
\text { pemetrexed }\end{array}$ \\
\hline TOP01 IHC - positive $(2+35 \%)$ & Irinotecan, topotecan & $\mathrm{n} / \mathrm{a}$ \\
\hline TOP2A IHC - positive $(2+30 \%)$ & $\begin{array}{l}\text { Doxorubicin, epirubicin, liposomal doxoru- } \\
\text { bicin }\end{array}$ & $\mathrm{n} / \mathrm{a}$ \\
\hline TUBB3 IHC - positive $(2+85 \%)$ & $\mathrm{n} / \mathrm{a}$ & $\mathrm{n} / \mathrm{a}$ \\
\hline TLE3 IHC - positive $(2+30 \%)$ & Docetaxel, nab-paclitaxel, paclitaxel & $\mathrm{n} / \mathrm{a}$ \\
\hline PGP IHC - negative & $\begin{array}{l}\text { Docetaxel, nab-paclitaxel, paclitaxel, doxo- } \\
\text { rubicin, epirubicin, liposomal } \\
\text { doxorubicin }\end{array}$ & $\mathrm{n} / \mathrm{a}$ \\
\hline ERCC1 IHC - negative & Carboplatin, cisplatin, oxaliplatin & $\mathrm{n} / \mathrm{a}$ \\
\hline MGMT IHC - positive $(1+45 \%)$ & & Dacabazine, temozolomide \\
\hline PD-L1 IHC positive $(2+5 \%)$ & Nivolumab, pembrolizumab & $\mathrm{n} / \mathrm{a}$ \\
\hline PD-1 IHC - positive (1/HPF) & Nivolumab, pembrolizumab & $\mathrm{n} / \mathrm{a}$ \\
\hline
\end{tabular}

